\title{
Complementary Analysis of Modal Content and Properties in a 19-cell Hollow Core Photonic Band Gap Fiber using Time-of- Flight and $\mathrm{S}^{2}$ Techniques
}

\author{
D.R. Gray, Z. Li, F. Poletti, R. Slavík, N.V. Wheeler, M.N. Petrovich, A. Obeysekara, and \\ D.J. Richardson \\ Optoelectronics Research Centre, University of Southampton, Southampton, SO171BJ, UK, \\ frap@orc.soton.ac.uk
}

\begin{abstract}
We study the rich multimode content of an ultra-low loss hollow core photonic bandgap fiber using two complementary techniques which allow us to investigate both short and long propagation distances. Several distinct vector modes are clearly identified, with evidence of low intermodal coupling and distributed scattering.
\end{abstract}

\section{Introduction}

Hollow Core Photonic Band Gap Fibers (HCPBGFs) show great promise for optical communications ${ }^{1}$ due to their ultralow optical nonlinearity, low latency and the potential for low transmission loss. Moreover, these properties make HC-PBGFs interesting for a host of other applications in sensing, gas based nonlinear optics and laser beam delivery, where the device length and loss requirements are often substantially less demanding.

In order to achieve the lowest losses and nonlinearities a large core is required, which is typically obtained by omitting 19 capillaries from a stacked preform - the resulting fibers being referred to as 19-cell HC-PBGFs. The downside of this is that it introduces a large number of core guided modes (indeed more than 40 vector modes are predicted, including all degeneracies) ${ }^{2}$.. This fact can significantly limit the usefulness of the fiber in many instances since the great majority of applications require stable transmission in a single spatial mode ideally the fundamental. To further complicate matters, surface modes can also propagate through $\mathrm{HC}-\mathrm{PBGFs}^{3}$ and their anti-crossings with the air guided modes of all orders increase the sensitivity of the fiber to external perturbations $^{4}$ and make stable mode propagation difficult ${ }^{5}$. This even makes optical mode reconstruction, which would otherwise be relatively straightforward through the use of the $\mathrm{S}^{2}$ or similar imaging techniques, very challenging ${ }^{6}$.

Recently we demonstrated a large core, 19cell HC-PBGF with no surface modes throughout the whole central part of the bandgap ${ }^{1}$. In that work we characterized the multimode content of the fiber (excited under different launch conditions) using a Time of Flight (ToF) technique, which enabled us to obtain a direct measurement of the Differential Group Delay (DGD) between different guided modes as well as an estimate of the amount of power transmitted into individual modes. This technique provides real time information but requires sufficient time delay between modes, hence long propagation lengths. Besides, it cannot provide information on what modes each observed peak corresponds to; this can only be estimated by comparison with simulations.

Here we apply a self interferometric technique $\left(S^{2}\right)$ to measure multimode content over short fiber lengths and simultaneously obtain the modal spatial distribution of intensity ${ }^{7}$. We find good agreement with our earlier ToF measurements as well as the expected evidence of additional high order mode peaks,
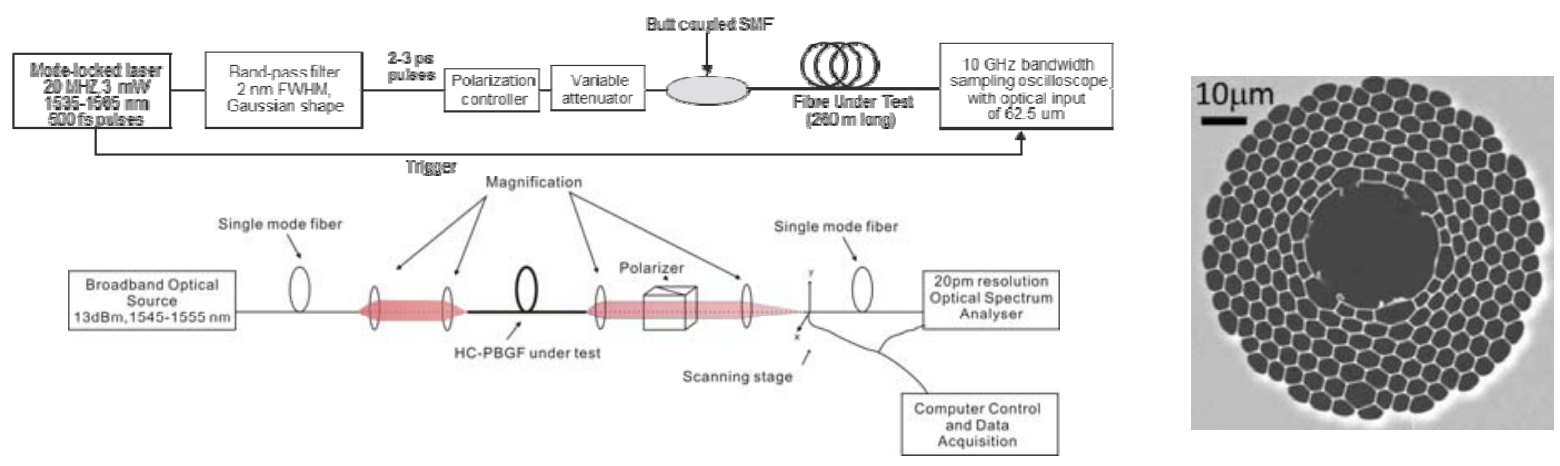

Fig. 1: Set-ups for time-of-flight (top) and $S^{2}$ (bottom) measurement, and SEM of the HC-PBGF under test. 


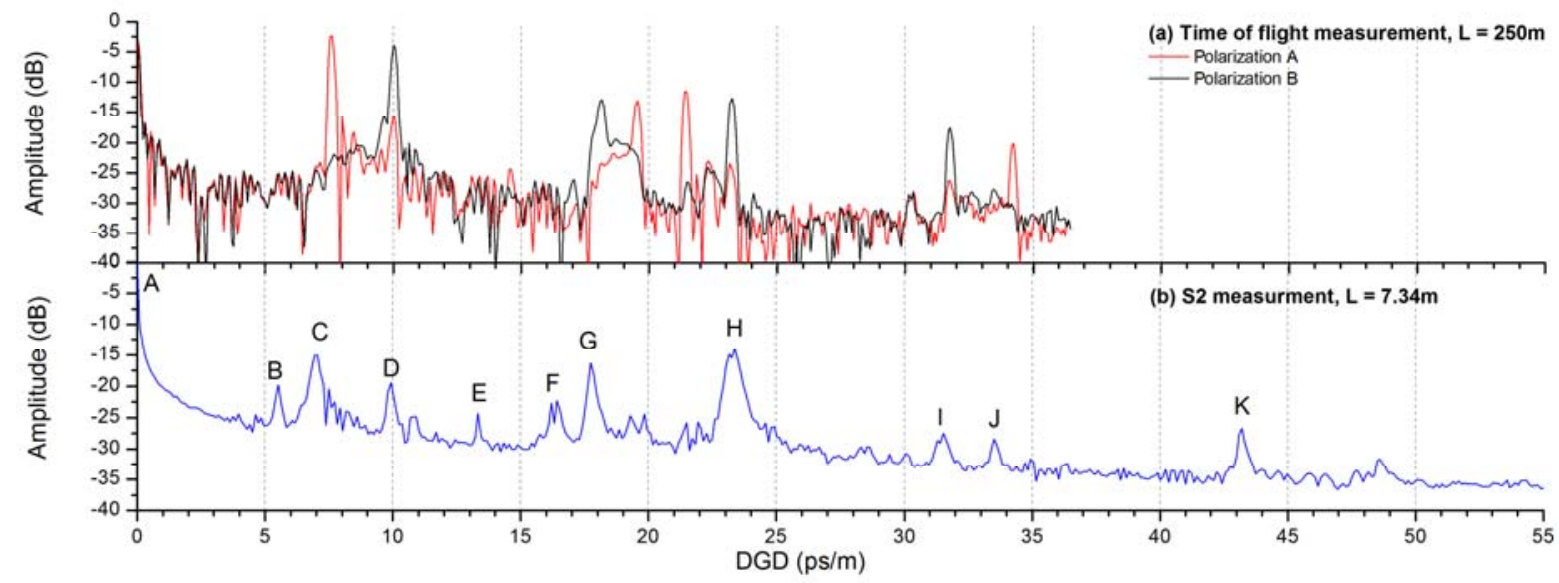

Fig. 2: Differential group delay (DGD) per unit length comparison between (a) ToF measurement over 250m of HC-PBGF; (b) $\mathrm{S}^{2}$ measurement over $7.34 \mathrm{~m}$ of the same fiber

which presumably disappear below the noise floor over the longer lengths used in our TOF measurements due to a higher propagation loss.

\section{Time of flight versus $\mathbf{S}^{2}$ set-ups}

Here we focus on the low loss, wide bandwidth HC-PBGF recently presented (see Fig. 1 for an SEM image) $)^{1}$. It has $61 / 2$ rings of cladding holes with an average pitch and relative hole size of $4.4 \mu \mathrm{m}$ and 0.97 respectively, and a core size of $26 \mu \mathrm{m}$. The fiber has a minimum loss of $3.5 \mathrm{~dB} / \mathrm{km}$ at $\sim 1500 \mathrm{~nm}$ and a very wide surface mode free central region of the bandgap with a 3-dB transmission bandwidth of $160 \mathrm{~nm}$.

Fig. 1 shows the two different set-ups used. In the time of flight set-up we launched 2-3 ps pulses emitted at $20 \mathrm{MHz}$ repetition rate from a passively mode-locked fiber laser into a $250 \mathrm{~m}$ long fiber and measured the corresponding temporal impulse response using a $10-\mathrm{GHz}$ bandwidth photodiode and fast sampling oscilloscope. The pulses were core-offset launched into the HC-PBGF to ensure efficient coupling to Higher Order Modes (HOMs). (For central coupling a clean fundamental mode was observed at the PBGF with power in the next highest intensity HOM reduced by $17 \mathrm{~dB}$ ).

In the $S^{2}$ set-up a low-coherence broadband Er:ASE source is used in combination with an Optical Spectrum Analyzer (OSA) to collect the spectrally resolved beatings between various modes at different spatial locations of the magnified near-field output of the fiber. A $7.34 \mathrm{~m}$ length of the HC-PBGF was used in this instance and the ASE light was free space launched (with a 1:1 imaging lens configuration) from a SMF (with a mode field diameter (MFD) of $9 \mu \mathrm{m}$ ) into the HC-PBGF (MFD 16 $\mu \mathrm{m}$ for the fundamental mode). The total measurement bandwidth was $10 \mathrm{~nm}(1545-1555 \mathrm{~nm})$ and the minimum nominal OSA $3-\mathrm{dB}$ resolution was
$20 \mathrm{pm}$, providing a maximum theoretical measurable group delay of $200 \mathrm{ps}(27.3 \mathrm{ps} / \mathrm{m}$ for this fiber length). In practice, we were able to resolve beatings with spectral periods smaller than the OSA 3-dB resolution, enabling us to reconstruct modes with $D G D$ values up to $43 \mathrm{ps} / \mathrm{m}$. Note that a polarizer was placed before the collection fiber in order to simplify the analysis of the results.

\section{Results}

In Fig. 2 we compare the results of the two measurements. From the ToF measurements (upper plot) 4 pairs of HOMs sited at around 8, 17,22 and $33 \mathrm{ps} / \mathrm{m}$ can clearly be identified. By modifying the input pulse polarization we were able to selectively excite either one or other of the two (polarization) modes associated with each HOM, or any linear combination of the two. Simulations suggest that the HOMs correspond to the $L P_{11}, L P_{21}, L P_{02}$ and $L P_{12}$ mode families, although there is no simple way to confirm this with this method alone. Other than these strong peaks that sit well above the $\sim-30 \mathrm{~dB}$ noise background there appears to be some evidence of other modes close to the noise floor but it is difficult to clearly resolve them.

The $S^{2}$ measurements (lower plot) broadly confirm the main features of the ToF measurement, with several peaks appearing at approximately the same value of DGD. The high intrinsic sensitivity of this interferometric technique and shorter fiber length used make it possible to clearly observe a larger number of peaks, even though no particular effort was made to excite HOMs by offsetting the free space launch. The inferred Multi Path Interference (MPI) is in some cases higher than $-50 \mathrm{~dB}$ illustrating the excellent dynamic range associated with this technique.

Fig. 3 shows the reconstructed modal 


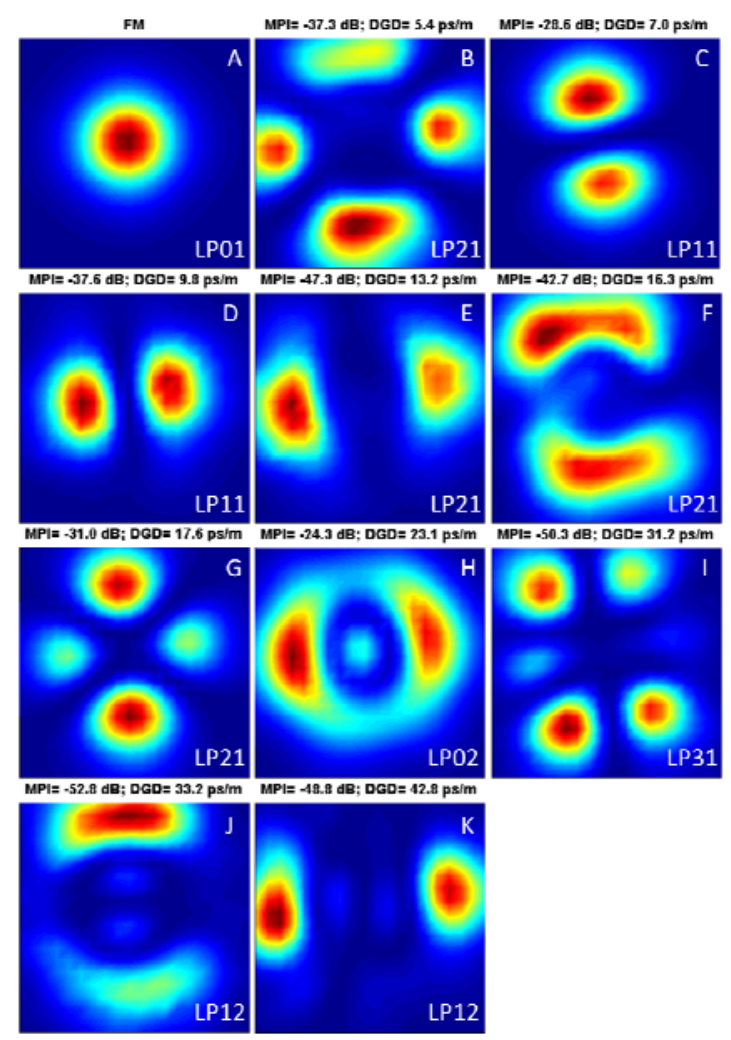

Fig. 3: Reconstructed intensities of the modes at peaks A-K in Fig.2. The respective MPI levels and DGDs are indicated above each figure

profiles of the 10 most prominent peaks in Fig. 2(b). All expected modes up to $\mathrm{LP}_{12}$ can be clearly distinguished, with some other interesting observations that can be made. For this particular launch there are 2 main $\mathrm{LP}_{11}$ peaks (C and $D$ ) each of which is followed by a smaller peak corresponding to a mode in the same spatial orientation but opposite polarization, all in the 7-11 ps/m region, in good agreement with the ToF results. Four distinct peaks corresponding to the 4 different vector modes of the $L P_{21}$ mode group (peaks $B, E, F$ and $G$ ), can also be observed although interestingly, mode $B$ seems to travel faster than the $\mathrm{LP}_{11}$ group, indicating unusual dispersive properties for this mode - possibly the result of an anti-crossing with a surface mode adjacent to the spectral region investigated here. For the $\mathrm{LP}_{02}$ mode (peak $\mathrm{H}$ ) only one of the two peaks seen with the ToF is observed in this particular measurement. In separate measurements (not shown here) we have however verified that a second peak around $21.5 \mathrm{ps} / \mathrm{m}$ can indeed be observed if the launch polarization is adjusted slightly. The peak at $32 \mathrm{ps} / \mathrm{m}$, erroneously attributed to the $\mathrm{LP}_{12}$ mode in previous work ${ }^{1}$, is actually the $\mathrm{LP}_{31}$ mode, while the two different peaks corresponding to orthogonally oriented $\mathrm{LP}_{12}$ modes can be seen to have a very broad separation (peaks $\mathrm{J}$ and $\mathrm{K}$ ), again likely evidence of unusual dispersive behavior.

It is important to emphasize that no plateau due to distributed scattering is evident after $7.34 \mathrm{~m}$ and the MPI in the region between peaks at around $12 \mathrm{ps} / \mathrm{m}$ is as low as $-58 \mathrm{~dB}$. Over a longer $250 \mathrm{~m}$ length the intermodal cross-talk is certainly below the $\sim 25 \mathrm{~dB}$ noise floor due of the photodiode impulse response. Such a low level of intermodal cross-talk and the well behaved modal content is promising for data transmission through reasonable lengths of such a fiber.

\section{Conclusions}

We have applied two very different techniques to provide modal analysis of a multimoded HCPBGF and generally observed good agreement in the results. Our results highlight the relative strengths of the two complementary approaches we have used: ToF measurements provides for real time MPI characterization over relatively long fiber lengths (such that the intermodal group delays are larger than $\sim 1 \mathrm{~ns}$ in this instance, although improved resolution is clearly possible using shorter pulses and a faster detection system). Although not shown here, the ToF technique also enables to measure the group velocity dispersion of the modes. $S^{2}$ is inherently a non-real-time technique (measurements typically take a few minutes) and it can only be used over relatively short fiber lengths as compared to ToF (the maximum total DGD has to be in the range of a few 100s to a few 1000s ps). However, the technique does allow for the simultaneous reconstruction of modal profiles, which in a complex scenario like in a HC-PBGF is extremely helpful. Our results indicate that robust single-mode data transmission should be achievable over at least $250 \mathrm{~m}$ of our current fiber (and probably over much longer lengths also when available) and that inter-mode coupling is also quite small illustrating potential for Space Division Multiplexing opportunities.

\section{Acknowledgements}

This work was supported by the European Communities $7^{\text {th }}$ Framework Programme under grant agreement 228033 (MODE-GAP).

\section{References}

[1] N. Wheeler et al, Proc OFC12, PDP5A.2.

[2] M. N. Petrovich et al., Opt. Express 16, 4337 (2008).

[3] C. M. Smith et al., Nature 424, 657 (2003)

[4] F. Poletti et al., Opt. Express 13, 9115 (2005)

[5] C. Peucheret et al. Electron. Lett. 41 (2004).

[6] A.M. DeSantolo et al., Proc CLEO11, CFM4.

[7] J. W. Nicholson et al., IEEE J. Sel. Top. Quant. Electron 15, 61 (2009). 